\title{
La covid-19: una carrera de fondo para avanzar en educación física
}

Covid-19: a long-distance race to advance in physical education

Francisco Javier Gil-Espinosa1

1Departamento de Didáctica de las Lenguas, las Artes y el Deporte, Universidad de Málaga, Andalucíatech, Málaga, España.

javiergil@uma.es

https://orcid.org/0000-0002-8845-4060

Detalles del artículo:

Número de palabras: 2.255; Tablas: 0; Figuras: 0; Referencias: 20

Recibido: julio 2020; Aceptado: agosto 2020; Publicado: septiembre 2020

Conflicto de interés: El autor declara que no existen conflictos de interés.

Correspondencia del autor: Francisco Javier Gil-Espinosa, javiergil@uma.es

\section{RESUMEN}

El 11 de marzo la Organización mundial de la salud (OMS) determina en su evaluación que la COVID-19 puede caracterizarse como una pandemia. Se justifica en los alarmantes niveles de propagación y su gravedad (OMS, 2020), de hecho, sólo en España, a fecha de 6 de junio de 2020 se han identificado 241.310 casos de infectados y 27.135 fallecidos (General y Sanidad, 2020). El dolor y daño personal y familiar se ha visto agravado con el cuestionamiento de algunos pilares de nuestra moderna sociedad, tales como los sistemas de prevención y atención de la salud y la globalización que, ante la demanda de productos sanitarios básicos, ha provocado una puja entre Gobiernos "amigos" por EPIS y respiradores.

Palabras clave: confinamiento, pandemia, actividad física. 


\section{ARTÍCULO DE OPINIÓN}

Con El 11 de marzo la Organización mundial de la salud (OMS) determina en su evaluación que la COVID-19 puede caracterizarse como una pandemia. Se justifica en los alarmantes niveles de propagación y su gravedad (OMS, 2020), de hecho, sólo en España, a fecha de 6 de junio de 2020 se han identificado 241.310 casos de infectados y 27.135 fallecidos (General y Sanidad, 2020). El dolor y daño personal y familiar se ha visto agravado con el cuestionamiento de algunos pilares de nuestra moderna sociedad, tales como los sistemas de prevención y atención de la salud y la globalización que, ante la demanda de productos sanitarios básicos, ha provocado una puja entre Gobiernos "amigos" por EPIS y respiradores.

\section{EFECTOS EN EL SISTEMA EDUCATIVO Y LA EDUCACIÓN FÍSICA}

Sin lugar a dudas, el confinamiento imprevisto se convirtió en un terremoto que ha sacudido nuestro sistema educativo (UNICEF, 2020), durante el cual, la mayoría del alumnado, familias y profesorado ha trabajado de forma rápida, incansable, ingeniosa y productiva. En contraste, se ha evidenciado una Administración lenta, desconocedora de muchas realidades y casuísticas de nuestro sistema y sus integrantes. Incapaz de tomar decisiones y disponer de recursos adecuados, ha recurrido a la autonomía de los centros educativos, delegando la responsabilidad en el profesorado y equipos directivos.

La situación sobrevenida, sin tiempo para la reflexión, junto con el cúmulo de variables a contemplar, como recursos y conocimientos de las familias, edad y nivel del alumnado, conocimiento tecnológico en el profesorado, así como su capacidad de adaptar y flexibilizar metodología y, en conjunto, todos los elementos del proceso de enseñanza-aprendizaje han supuesto un enorme reto para el desempeño en todas las asignaturas del currículo.

La actividad física (AF) regular tiene numerosas implicaciones no sólo para la salud física, psicológica y social de los niños (Alvarez-Pitti et al., 2020; Pascoe et al., 2020), sino también para el logro y rendimiento académico (Gil-Espinosa et al., 2019), de hecho, la condición física en general y, la capacidad cardiovascular en particular, es una poderosa herramienta de salud física y mental (Lang et al., 2018; Ortega et al., 2008). Igualmente, la práctica regular de actividad física mejora el sistema inmunológico (Fernandez, Clemente, y Giannarelli, 2018; López Marcos, 2014). Es posible que, motivado en estos argumentos, un sector del profesorado, preocupado por el bienestar de su alumnado y ante la emergencia y premura de la situación, decidió trabajar en propuestas muy orientadas a contenidos de condición física debido a la inactividad que podría provocar el confinamiento en casa (Cruz et al., 2020; Mera et al., 2020). El reto ha sido enorme y, sólo tras superar una primera fase de noqueo, el colectivo de profesionales comenzó a reflexionar y tomar decisiones ajustadas a las necesidades de la asignatura, como ocurrió con otras muchas materias curriculares.

En este contexto, nos encontramos la asignatura de Educación Física cuya finalidad principal es desarrollar en las personas su competencia motriz, entendida como la integración de los conocimientos, los procedimientos, las actitudes y los sentimientos vinculados a la conducta motora fundamentalmente. Asimismo, está vinculada a la adquisición de competencias relacionadas con la salud través de acciones que ayuden a la adquisición de hábitos responsables de actividad física regular, y de la adopción de actitudes críticas ante prácticas sociales no saludables (Real Decreto 126, 2014). De hecho, entre las razones por las 
que la EF debe llegar a todos los jóvenes además de cuestiones como promover el bienestar, prevenir lesiones, luchar contra la obesidad también se encuentran otras como proporcionar oportunidades únicas de actividades, de habilidades motoras y ayudar a la educación integral (Le Masurier y Corbin, 2006). Por tanto, la Educación Física engloba la enseñanza-aprendizaje de otras esferas del alumnado, si bien, su competencia motriz es la principal finalidad, junto a ella se promueve un desarrollo integral que enriquece individual y socialmente (Gil Espinosa y Aznar Cebamanos, 2016; UNICEF-ONU-UNESCO, 2015).

Aunque se han registrado en redes sociales y televisión muchas propuestas de actividad física orientadas exclusivamente a la mejora de la condición física, otras muchas iniciativas del profesorado han contemplado todos los aspectos curriculares, procurando un aprendizaje competencial, sin olvidar ninguna de las situaciones motrices o bloques de contenidos (Burgueño et al., 2020a; Burgueño et al., 2020b). Igualmente, ha sido un momento para avanzar en metodologías innovadoras, individualizadas y adaptadas a cada contexto familiar. A su vez, han proliferado propuestas basadas en retos, en proyectos educativos, integradoras de varios contenidos o situaciones motrices (intradisciplinares) y, en otros casos, integrando aspectos de varias asignaturas (interdisciplinares) bajo el paraguas del uso de las tecnologías de la información y comunicación (TIC) en su esfera más avanzada, convertidas en tecnologías para el aprendizaje y el conocimiento (TAC)

En cualquier caso, bajo mi punto de vista, posiblemente hayamos tenido la mejor oportunidad para trabajar dos cuestiones imprescindibles para la consecución de una EF de CAlidad y Éxito (EFCAE), como son el desarrollo de la autonomía en el alumnado y la participación familiar, no solo en el seguimiento y guía de las actividades sino también en la realización conjunta de las mismas.

Otra cuestión para la reflexión es el rol docente, inevitablemente ha tenido que ser modificado. Posiblemente ha sido el periodo de mayor utilización de estilos de enseñanza productivos, de indagación y búsqueda. A su vez, dentro de los estilos reproductivos, los individuales habrán protagonizado la atención a la diversidad, en esta ocasión, añadiendo variables como el tipo de vivienda y la disponibilidad de medios tecnológicos o de otro tipo. Sin lugar a dudas, el aprendizaje en el profesorado de las TAC supondrá un punto de inflexión que debería tener consecuencias positivas en el futuro del proceso de enseñanza-aprendizaje de la Educación Física.

\section{Y A PARTIR DE AHORA ¿QUÉ?}

Sin entrar a valorar los posibles escenarios en los que tendremos que desempeñar la enseñanza-aprendizaje de la EF en el próximo curso deberíamos recopilar todas las buenas experiencias y aprendizajes que nos ha permitido esta situación de docencia no presencial. Sin lugar a dudas, la docencia presencial de la EF es insustituible para lograr unos altos niveles de calidad, pero avanzar en el uso de las nuevas tecnologías para el aprendizaje, la implementación de metodologías más activas donde se empodere al alumnado y se implique a las familias, nos permitirá alcanzar estándares de competencia motora, para la salud y calidad de vida que no conseguiremos de otra forma.

Es triste oír y leer, a responsables políticos, hablar de utilizar los espacios dedicados a la docencia de la Educación Física como "aulas". Sin lugar a dudas, se muestra un gran 
desconocimiento del sistema educativo, de la asignatura y sus beneficios en el desarrollo individual y social. Resulta paradójico, justificar esa medida para preservar la salud de los estudiantes cuando la OMS ya declaró la "obesidad" como pandemia, también porque España presenta unos de los datos más preocupantes en "obesidad infantil" del mundo. La OMS (2020) establece que un elevado índice de masa corporal se constituye en un factor de riesgo para enfermedades no transmisibles como las cardiovasculares (principalmente las cardiopatías y los accidentes cerebrovasculares), la diabetes, los trastornos del aparato locomotor (en especial la osteoartritis, una enfermedad degenerativa de las articulaciones), y algunos cánceres. A su vez, apunta que la obesidad infantil se asocia con una mayor probabilidad de obesidad, muerte prematura y discapacidad en la edad adulta. Sin embargo, además de estos mayores riesgos futuros, los niños obesos sufren dificultades respiratorias, mayor riesgo de fracturas e hipertensión, y presentan marcadores tempranos de enfermedades cardiovasculares, resistencia a la insulina y efectos psicológicos. Recomendando la propia OMS (2020), como medida para prevenir la obesidad y el sobrepeso, realizar una actividad física periódica de 60 minutos diarios para los jóvenes y 150 minutos semanales para los adultos.

Resulta inexplicable, por qué las Administraciones no han atendido a la pandemia de la obesidad en concordancia con su gravedad para la salud. Especialmente la educativa, porque es la única que, integrando la Educación Física en sus currículos obligatorios puede garantizar que se llega a todos los niños y jóvenes, independiente de su nivel socio-cultural y económico. Téngase en consideración que, los hijos de familias con niveles socio-educativo-culturales más bajos, son quienes mantienen una peor alimentación y más bajos niveles de actividad física y deportiva. Incluso existen estudios que recomiendan un incremento del tiempo de Educación Física en los centros educativos para mejorar los resultados académicos del alumnado más desfavorecido socialmente (Gil-Espinosa et al., 2020).

El Consejo General de la Educación Física y Deportiva (2020) ha publicado unas recomendaciones docentes para una Educación Física escolar segura y responsable ante la nueva normalidad donde se contemplan dos escenarios. El objetivo es minimizar los riesgos de contagio por la COVID-19 en las clases de EF, donde se recomienda usar espacios al aire libre, priorizar materiales fácilmente des infectables, evitar materiales compartidos, junto a otras propuestas técnicas y profesionalmente diseñadas.

Los profesionales de la Educación Física actuarán con criterios de SALUD, ¡sí, en mayúsculas! Es decir, minimizando el máximo los riesgos de contagio por COVID-19 pero, también luchando contra otra pandemia, la obesidad.

A la vez, continuaremos trabajando por una formación integral de nuestros jóvenes con la clara intencionalidad de que revierta en la sociedad. Esperemos que un futuro próximo, dicha apuesta por la formación integral se vea reflejada en la gestión política de nuestra sociedad, sus retos y desafíos.

Cualquier logro y avance para la Educación Física ha conllevado mucho esfuerzo. Para ser atendidas se han tenido que justificar con numerosas evidencias científicas. Por tanto, en este difícil momento, tenemos que continuar trabajando con fundamentación científica y con pro de la mejora de nuestro alumnado y sociedad. 


\section{REFERENCIAS}

Alvarez-Pitti, J., Casajús Mallén, J. A., Leis Trabazo, R., Lucía, A., López de Lara, D., Moreno Aznar, L. A., y Rodríguez Martínez, G. (2020). Exercise as medicine in chronic diseases during childhood and adolescence. Anales de Pediatria, 92(3), 173.e1-173.e8. https://doi.org/10.1016/j.anpedi.2020.01.010

Burgueño, R.; Espejo, R.; López-Fernández, I.; Gil-Espinosa, F. J. (2020a). EDUCACIÓN FÍSICA DE CALIDAD EN CASA PARA NIÑOS: UNA PROPUESTA DE APLICACIÓN CURRICULAR EN EDUCACIÓN PRIMARIA. Journal of Sport and Health Research., 12(2), 270-287. Recuperado de https://recyt.fecyt.es/index.php/JSHR/article/view/80564

Burgueño, R., Bonet-Medina, A., Cerván-Cantón, A., Espejo, R., Fernández-Berguillo, F.B., Gordo-Ruiz, F., Linares-Martínez, H., Montenegro-Lozano, S., Ordoñez-Tejero, N., Vergara-Luque, J.J., y Gil-Espinosa, F.J. (2020b). Educación Física en Casa de Calidad. Propuesta de aplicación curricular en Educación Secundaria Obligatoria. Quality Physical Education at Home. Curricular implementation proposal in Middle Secondary. RETOS, 2021.

Consejo General de la Educación Física y Deportiva (2020). Recomendaciones docentes para una Educación Física escolar segura y responsable ante la nueva normalidad. Consejo COLEF. https://www.consejocolef.es/post/covid19-guia-ef-nueva-normalidad

Organización Mundial de la Salud (2020). COVID-19: cronología de la actuación de la OMS. 4-7. Recuperado de https://www.who.int/es/news-room/detail/27-04-2020-who-timeline---covid-19

Cruz, L., Betancourt, M., Fonseca, I., Bernate, J. A., Arias, C., y Gómez, K. (2020). Viref Revista de Educación Física. 63-71.

España. RD 126/2014, de 28 de febrero. Curriculum Básico Ed. Primaria. Boletín Oficial del Estado, 19349-19420. Recuperado de http://www.boe.es/boe/dias/2014/03/01/pdfs/BOE-A-2014-2222.pdf

Fernandez, D. M., Clemente, J. C., y Giannarelli, C. (2018). Physical activity, immune system, and the microbiome in cardiovascular disease. Frontiers in Physiology. https://doi.org/10.3389/fphys.2018.00763

General, S., y Sanidad, D. E. (2020). Situación en españa.

Gil-Espinosa, F. J., Cadenas-Sanchez, C., y Chillón, P. (2019). Physical fitness predicts the academic achievement over one-school year follow-up period in adolescents. Journal of Sports Sciences, 37(4). https://doi.org/10.1080/02640414.2018.1505184

Gil-espinosa, F. J., Chill, P., y Fern, C. (s. f.). Association of Physical Fitness with Intelligence and Academic Achievement in Adolescents.

Gil Espinosa, F., y Aznar Cebamanos, M. (2016). Educación física de calidad en el sistema educativo español. Revista Española de Educación Física y Deportes: REEFD.

Lang, J. J., Belanger, K., Poitras, V., Janssen, I., Tomkinson, G. R., y Tremblay, M. S. (2018). Systematic review of the relationship between $20 \mathrm{~m}$ shuttle run performance and health indicators among children and youth. Journal of Science and Medicine in Sport. https://doi.org/10.1016/j.jsams.2017.08.002

Le Masurier, G., y Corbin, C. B. (2006). Top 10 Reasons for Quality Physical Education. Journal of Physical Education, Recreation y Dance. https://doi.org/10.1080/07303084.2006.10597894

López Marcos, J. J. (2014). Efectos de la Actividad Física sobre el Sistema Inmune | Blog EMP. 6(1), 74-80. Recuperado de https://blog.medicapanamericana.com/efectos-de-la-actividad-fisica-sobre-el-sistemainmune/\%0Ahttp://blog.medicapanamericana.com/efectos-de-la-actividad-fisica-sobre-el-sistemainmune/

Mera, A. Y., Tabares-Gonzalez, E., Montoya-Gonzalez, S., Muñoz-Rodriguez, D. I., y Monsalve Vélez, F. (2020). Recomendaciones prácticas para evitar el desacondicionamiento físico durante el confinamiento por pandemia asociada a COVID-19. Universidad y Salud. https://doi.org/10.22267/rus.202202.188

Ortega, F. B., Ruiz, J. R., Castillo, M. J., y Sjöström, M. (2008). Physical fitness in childhood and adolescence: A powerful marker of health. International Journal of Obesity, Vol. 32, pp. 1-11. https://doi.org/10.1038/sj.ijo.0803774

Pascoe, M., Bailey, A. P., Craike, M., Carter, T., Patten, R., Stepto, N., y Parker, A. (2020). Physical activity and exercise in youth mental health promotion: A scoping review. BMJ Open Sport and Exercise Medicine. https://doi.org/10.1136/bmjsem-2019-000677

UNICEF (2020). Al Covid-19 Frenar El Virus. Recuperado de https://www.unicef.es/educa/biblioteca/laeducacion-frente-al-covid-19

UNICEF-ONU-UNESCO. (2015). Educación física de calidad - Guía para los responsables políticos. En Unesco. 